\begin{tabular}{|c|l|}
\hline Title & A time like surface in Minkowski 3-space which contains light-like lines \\
\hline Author(s) & Izumiya, S.; Takiyama, A. \\
\hline Citation & Hokkaido University Preprint Series in Mathematics, 272, 1-7 \\
\hline Issue Date & 199412-1 \\
\hline DOI & 10.14943/83419 \\
\hline Doc URL & http://hdl.handle.net/2115/69023 \\
\hline Type & bulletin (article) \\
\hline File Information & pre272.pdf \\
\hline
\end{tabular}

Instructions for use 
A TIME-LIKE SURFACE IN MINKOWSKI 3-SPACE WHICH CONTAINS LIGHT-LIKE LINES

S. Izumiya and A. Takiyama

Series $\sharp 272$. December 1994 


\section{HOKKAIDO UNIVERSITY \\ PREPRINT SERIES IN MATHEMATICS}

$\sharp 246$ T. Nakazi, M. Yamada, $\left(\mathrm{A}_{2}\right)$-Conditions and Carleson Inequalities, 27 pages. 1994.

$\sharp 247$ N. Hayashi, K. Kato and T. Ozawa, Dilation Method and smoothing Effect of Solutions to the Benjamin-ono Equation, 17 pages. 1994.

\#248 H. Kikuchi, Sheaf cohomology theory for measurable spaces, 12 pages. 1994.

$\sharp 249$ A. Inoue, Tauberian theorems for Fourier cosine transforms, 9 pages. 1994.

$\sharp 250$ S. Izumiya, G. T. Kossioris, Singularities for viscosity solutions of Hamilton-Jacobi equations, 23 pages. 1994.

$\sharp 251$ H. Kubo, K. Kubota, Asymptotic behaviors of radially symmetric solutions of $\square u=|u|^{p}$ for super critical values $p$ in odd space dimensions, 51 pages. 1994.

\#252 T. Mikami, Large Deviations and Central Limit Theorems for Eyraud-Farlie-Gumbel-Morgenstern Processes, 9 pages. 1994.

$\sharp 253$ T. Nishimori, Some remarks in a qualitative theory of similarity pseudogroups, 19 pages. 1994.

$\sharp 254$ T. Suwa, Residues of complex analytic foliations relative to singular invariant subvarieties, 15 pages. 1994.

$\sharp 255 \quad$ T. Tsujishita, On Triple Mutual Information, 7 pages. 1994.

$\sharp 256$ T. Tsujishita, Construction of Universal Modal World based on Hyperset Theory, 15 pages. 1994.

\#257 A. Arai, Trace Formulas, a Golden-Thompson Inequality and Classical Limit in Boson Fock Space, 35 pages. 1994.

$\sharp 258$ Y-G. Chen, Y. Giga, T. Hitaka and M. Hon ma, A Stable Difference Scheme for Computing Motion of Level Surfaces by the Mean Curvature, 18 pages. 1994.

$\sharp 259 \quad$ K. Iwata, J. Schäfer, Markov property and cokernels of local operators, 7 pages. 1994.

$\sharp 260$ T. Mikami, Copula fields and its applications, 14 pages. 1994.

$\sharp 261$ A. Inoue, An Abel-Tauber theorem for Fourier sine transforms, 6 pages. 1994.

$\sharp 262$ N. Kawazumi, Homology of hyperelliptic mapping class groups for surfaces, 13 pages. 1994.

$\sharp 263$ Y. Giga, M. E. Gurtin, A comparison theorem for crystalline evolution in the plane, 14 pages. 1994.

$\sharp 264$ J. Wierzbicki, On Commutativity of Diagrams of Type $\Pi_{1}$ Factors, 26 pages. 1994.

$\sharp 265$ N. Hayashi, T. Ozawa, Schrödinger Equations with nonlinearity of integral type, 12 pages. 1994.

$\sharp 266$ T. Ozawa, On the resonance equations of long and short waves, 8 pages. 1994.

\$267 T. Mikami, A sufficient condition for the uniqueness of solutions to a class of integro-differential equations, 9 pages. 1994.

$\sharp 268$ Y. Giga, Evolving curves with boundary conditions, 10 pages. 1994.

\#269 A. Arai, Operator-theoretical analysis of representation of a supersymmetry algebra in Hilbert space, 12 pages. 1994.

$\sharp 270$ A. Arai, Gauge theory on a non-simply-connected domain and representations of canonical commutation relations, 18 pages. 1994.

$\sharp 271$ S. Jimbo, Y. Morita and J. Zhai, Ginzburg landau equation and stable steady state solutions in a non-trivial domain, 17 pages. 1994 . 


\title{
A TIME-LIKE SURFACE IN MINKOWSKI 3-SPACE WHICH CONTAINS LIGHT-LIKE LINES
}

\author{
Shyuichi Izumiya and Akihiro Takiyama
}

Simple characterizations of a pseudosphere or a time-like plane in Minkowski 3-space by the existence of light-like lines are given.

\section{INTRODUCTION}

There are many simple characterizations of a sphere in Euclidean 3-space [1,3,4]. For example "all geodesics are plane curves" characterizes a sphere or a plane. In [4] Takeuchi gave a much simpler and practical characterization of a sphere. Her characterization is as follows:if there exist four geodesics through each point of a complete surface such that they are plane curves, then the surface is a sphere or a plane. She also gave other characterizations of a sphere or a plane in [4].

In this paper we consider the similar problem for a pseudosphere $S_{1}^{2}(r, a)$ (for definition, see Section 2) and a time-like plane in Minkowski 3-space. The normal vector field on a pseudosphere in $\mathbb{M}^{3}$ is light-like. We say that a surface $S$ in $\mathbb{M}^{3}$ is time-like if the normal vector field on $S$ is space-like. It is easy to show that if all geodesics on a time-like surface are plane curves then the surface is an open subset of a pseudosphere or a time-like plane in Minkowski 3-space $\mathbb{M}^{3}$ (cf., [5]). We give characterizations which are much simpler and peculiar to the case for a pseudosphere or a time-like plane in Minkowski 3-space $\mathbb{M}^{3}$. Our main results are as follows.

THEOREM A. Let $S$ be a connected time-like surface in Minkowski 3-space $\mathbb{M}^{3}$. For each point $p$ of $S$, suppose that there exist two light-like curves on $S$ through $p$ such that they are plane curves. Then $S$ is a open subset of a pseudosphere or a time-like plane. 
We have corollaries of the theorem as follows:

COROLLARY A.1. Let $S$ be a time-like surface in Minkowski 3-space $\mathbb{M}^{3}$. For each point $p$ of $S$, suppose that the intersection of $S$ and $T_{p} S$, which is considered as an affine plane in $\mathbb{M}^{3}$, is the two lines such that each direction is light-like. Then $S$ is a pseudosphere.

The assumption of the corollary can be interpreted as that if two lines can be pressed entirely at a point $p$ of the surface, then these are the light cone on the tangent plane.

We have a simple characterization of a time-like plane in $\mathbb{M}^{3}$ as an corollary of Theorem A.

COROLLARY A.2. Let $S$ be a connected time-like surface in $\mathbb{M}^{3}$. For each point $p$ of $S$, suppose that there exist two light-like curves of $S$ which are plane curve and one non-light-like line on $S$ through $p$. Then $S$ is a open set of a time-like plane.

Another characterization by the existence of planar geodesics is given by the following.

THEOREM B. Let $S$ be a connected time-like surface in Minkowski 3-space $\mathbb{M}^{3}$. For each point $p$ in $S$, suppose that there exist one light-like curve and two non-light-like geodesics on $S$ through $p$ such that they are plane curves. Then $S$ is an open subset of a pseudosphere or a time-like plane.

We also have the following simple characterization of a time-like plane in $\mathbb{M}^{3}$ as a corollary of Theorem B.

COROLLARY B.1. Let $S$ be a connected time-like surface in Minkowski 3-space $\mathbb{M}^{3}$. For each point $p$ in $S$, suppose that there exist one light-like curve, one non-light-like geodesics on $S$ such that they are plane curves and one non-light-like line through $p$. Then $S$ is an open subset of a time-like plane.

We remark that these theorems are the best possible in some sense (cf., Section 4). For space-like surfaces, the induced metric on the surface is positively definite, so that we can give characterizations of a hyperbolic surface $H_{1}^{2}(r, a)$ by exactly the same arguments as those in $[1,3,4]$. Thus we do not consider this case in this paper.

All surfaces and maps considered here are of class $C^{\infty}$ unless stated otherwise.

\section{BASIC NOTIONS}

Let $\mathbb{R}^{3}=\left\{\left(x_{1}, x_{2}, x_{3}\right) \mid x_{1}, x_{2}, x_{3} \in \mathbb{R}\right\}$ be the usual oriented 3-dimensional vector space and differential manifold, which is oriented by $e_{1}=(1,0,0), e_{2}=(0,1,0)$ and $e_{3}=$ $(0,0,1)$ and given the Euclidean differentiable structure. Minkowski 3 -space is defined by $\mathbb{M}^{3}=\left\{\mathbb{R}^{3}, I_{(2,1)}\right\}$, where $I_{(2,1)}=d x_{1}^{2}+d x_{2}^{2}-d x_{3}^{2}$. Thus the metric tensor is given by 
$<\mathrm{X}, \mathrm{Y}>=x_{1} y_{1}+x_{2} y_{2}-x_{3} y_{3}$, where $\mathrm{X}=\left(x_{1}, x_{2}, x_{3}\right)$ and $\mathrm{Y}=\left(y_{1}, y_{2}, y_{3}\right)$. A vector $\mathrm{X}$ in $\mathbb{M}^{3}$ is called light-like if $\langle\mathbf{X}, \mathbf{X}\rangle=0$, space-like if $\langle\mathbf{X}, \mathbf{X}>>0$ and time-like if $<\mathrm{X}, \mathrm{X}><0$. A curve $\gamma$ is called light-like if its tangent vector field $\gamma^{\prime}$ is always light-like. We also say that a curve $\gamma$ is non-light-like if its tangent vector field $\gamma^{\prime}$ is always space-like or time-like. The pseudosphere is defined to be

$$
S_{1}^{2}(r, a)=\left\{\left(x_{1}, x_{2}, x_{3}\right) \mid\left(x_{1}-a_{1}\right)^{2}+\left(x_{2}-a_{2}\right)^{2}-\left(x_{3}-a_{3}\right)^{2}=r^{2}\right\},
$$

where $a=\left(a_{1}, a_{2}, a_{3}\right)$ is the center and $r>0$ is the radial of $S_{1}^{2}(r, a)$.

The Levi-Civita connection of $\mathbb{M}^{3}$ is denoted by $\nabla$. Let $S$ be a surface in $\mathbb{M}^{3}$. We say that $S$ is time-like if the normal vector field to $S$ is space-like. Let $\Pi(\mathrm{X}, \mathrm{Y})$ be a second fundamental form tensor of $S$ in $\mathbb{M}^{3}$. Since $S$ is a time-like surface, there exists (at least locally) a unit normal vector field $\xi$ on $S$. We have the following formula:

$$
<\Pi(\mathrm{X}, \mathrm{Y}), \xi>=<-\nabla \mathbf{X} \xi, \mathrm{Y}>.
$$

If we denote that $\Pi(\mathbf{X}, \mathbf{Y})=\sigma(\mathbf{X}, \mathbf{Y}) \xi$, we have $\sigma(\mathbf{X}, \mathbf{Y})=\langle-\nabla \mathbf{X} \xi, \mathbf{Y}\rangle$, so that $-\nabla_{\mathbf{X}} \xi$ is the shape operator in this case It is well-known that the shape operator is self-adjoint with respect to $<,>$ (i.e., $\left.<-\nabla_{\mathbf{X}} \xi, \mathrm{Y}\right\rangle=\left\langle\mathbf{X},-\nabla_{\mathbf{Y}} \xi>\right.$ ).

We now state some lemmas for preparing the proof of main results.

LEMMA 2.1 Let $V$ be a time-like plane in $\mathbb{M}^{3}$. If $\langle\mathrm{X}, \mathrm{X}\rangle=0$ and $\langle\mathrm{Y}, \mathrm{Y}\rangle \neq 0$, then $<\mathrm{X}, \mathrm{Y}>\neq 0$.

Proof. Since $V$ has two light-like direction, there exists $\mathrm{Z} \in V$ such that $\langle\mathrm{Z}, \mathrm{Z}\rangle=0$ and $\mathbf{Z} \notin\langle\mathbf{X}\rangle_{\mathbb{R}}$. It follows that there exist real numbers $\lambda, \mu$ with $\mu \neq 0$ such that $\mathbf{Z}=\lambda \mathbf{X}+\mu \mathbf{Y}$. Thus we have

$$
\left.0=<\mathrm{Z}, \mathrm{Z}>=2 \lambda \mu<\mathrm{X}, \mathrm{Y}>+\mu^{2}<\mathrm{Y}, \mathrm{Y}\right\rangle \text {. }
$$

If $\langle\mathrm{X}, \mathrm{Y}\rangle=0$, then $\langle\mathrm{Y}, \mathrm{Y}\rangle=0$. This is a contradiction

Since the light direction on a time-like plane in Minkowski 3-space $\mathbb{M}^{3}$ is constant, we have the following simple lemma.

LEMMA 2.2 Let $\gamma$ be a light-like plane curve in $\mathbb{M}^{3}$. Then it is a line, especially a geodesic in $\mathbb{M}^{3}$.

We also have the following lemma for light-like line on a surface in $\mathbb{M}^{3}$.

LEMMA 2.3 Let $\gamma$ be a light-like line on a time-like surface in $\mathbb{M}^{3}$ and $\xi$ be a normal unit vector field on $S$. Then there exists an real number $k$ such that $-\nabla_{\mathbf{X}} \xi=k \mathrm{X}$, where $\mathrm{X}$ is a tangent vector of $\gamma$. 
Proof. Since $\gamma$ is light-like line, we have $\sigma(\mathbf{X}, \mathbf{X})=0$ (cf., [2], page 103 Corollary 9). It follows that

$$
<-\nabla_{\mathbf{X}} \xi, \mathbf{X}>=\sigma(\mathbf{X}, \mathbf{X})=0
$$

Suppose that there are no real numbers $k$ such that $-\nabla \mathbf{x} \xi=k \mathrm{X}$. Since $\operatorname{dim} S=2$, for any $\mathbf{Y} \in T_{p} S$, there exist real numbers $\lambda, \mu$ such that $Y=\lambda(-\nabla \mathbf{x} \xi)+\mu k \mathbf{X}$. Thus we have

$$
<\mathrm{X}, \mathrm{Y}>=\lambda<-\nabla_{\mathrm{X}} \xi, \mathrm{X}>+\mu<\mathrm{X}, \mathrm{X}>=0 \text {. }
$$

Let $\mathrm{Y}$ be a tangent vector at $p$ which has another light-like direction. Then we have

$$
\begin{aligned}
\langle\mathrm{X}+\mathrm{Y}, \mathrm{X}+\mathrm{Y}\rangle & =\langle\mathrm{X}, \mathrm{X}\rangle+2\langle\mathrm{X}, \mathrm{Y}\rangle+\langle\mathrm{Y}, \mathrm{Y}\rangle \\
& =2\langle\mathrm{X}, \mathrm{Y}\rangle=0,
\end{aligned}
$$

This contradicts to the fact that $\mathrm{X}+\mathrm{Y}$ is not light-like.

Let $\gamma$ be a non-light-like curve. We may assume that $\gamma$ is parametrized by arc length $s$. Thus we have $\left\langle\gamma^{\prime}(s), \gamma^{\prime}(s)\right\rangle=\varepsilon(s)= \pm 1$. By the exactly same arguments as in the case for a curve in Euclidean 3-space, we have the following Frenet-Serret formula (cf., [5]): There exist field of vectors $\mathbf{n}(s), \mathbf{b}(s)$ with $\langle\mathbf{n}(s), \mathbf{n}(s)>=\delta(s)= \pm 1,\langle\mathbf{b}(s), \mathbf{b}(s)>=-\varepsilon(s) \delta(s)$ and functions $k=k(s), \tau=\tau(s)$ along $\gamma$ such that

$$
\begin{aligned}
& \nabla_{\gamma^{\prime}(s)} \gamma^{\prime}(s)=k(s) \mathrm{n}(s) \\
& \nabla_{\gamma^{\prime}(s)} \mathrm{n}(s)=-\varepsilon(s) \delta(s) k(s) \gamma^{\prime}(s)+\varepsilon(s) \tau(s) \mathrm{b}(s) \\
& \nabla_{\gamma^{\prime}(s)} \mathrm{b}(s)=\tau(s) \mathrm{n}(s) .
\end{aligned}
$$

If $\gamma$ is a plane curve, then $\tau(s) \equiv 0$, so that we have

$$
\nabla_{\gamma^{\prime}(s)} \gamma^{\prime}(s)=k(s) \mathbf{n}(s) \text { and } \nabla_{\gamma^{\prime}(s)} \mathbf{n}(s)=-\varepsilon(s) \delta(s) k(s) \gamma^{\prime}(s)
$$

Suppose that $\gamma$ is also a geodesic of $S$, then

$$
\nabla_{\gamma^{\prime}(s)} \gamma^{\prime}(s)=\Pi\left(\gamma^{\prime}(s), \gamma^{\prime}(s)\right)=\sigma\left(\gamma^{\prime}(s), \gamma^{\prime}(s)\right) \xi(\gamma(s))
$$

(cf., [2], page 103 Corollary 9). It follows that $\mathrm{n}(s)= \pm \xi(\gamma(s))$, so we have the following lemma.

LEMMA 2.4 Let $S$ be a time-like surface in Minkowski 3-space $\mathbb{M}^{3}$ and $\gamma$ be a non-lightlike geodesic on $S$ which is parametrized by arc length $s$ such that it is a plane curve. Then $\sigma\left(\gamma^{\prime}(s), \gamma^{\prime}(s)\right)=0$ when $k(s)=0$ and $\nabla_{\gamma^{\prime}(s)} \xi(\gamma(s))=\mp \varepsilon(s) \delta(s) k(s) \gamma^{\prime}(s)$ when $k(s) \neq 0$.

\section{PROOF OF RESULTS}

We now give a proof of Theorem $\mathrm{A}$. 
Proof of Theorem A. Let $\gamma_{i}(i=1,2)$ be two light-like curve on $S$ through $p$ such that they are plane curves. By Lemma 2.3, we have $-\nabla_{\mathbf{X}_{i}} \xi=k_{i} \xi$ for some $k_{i}$, where $\mathrm{X}_{i}$ is the tangent vector of $\gamma_{i}(i=1,2)$. If $k_{1} \neq k_{2}$, we have

$$
\begin{aligned}
& k_{1}<\mathbf{X}_{1}, \mathbf{X}_{2}>=<k_{1} \mathbf{X}_{1}, \mathbf{X}_{2}>=<-\nabla \mathbf{X}_{1} \xi, \mathbf{X}_{2}> \\
& =<\mathbf{X}_{1},-\nabla \mathbf{X}_{2} \xi>=<\mathbf{X}_{1}, k_{2} \mathbf{X}_{2}>=k_{2}<\mathbf{X}_{1}, \mathbf{X}_{2}>
\end{aligned}
$$

so that $\left\langle\mathbf{X}_{1}, \mathbf{X}_{2}\right\rangle=0$.

Since $\mathrm{X}_{1}+\mathrm{X}_{2}$ is not light-like vector, we have

$0 \neq<\mathrm{X}_{1}+\mathrm{X}_{2}, \mathrm{X}_{1}+\mathrm{X}_{2}>=<\mathrm{X}_{1}, \mathrm{X}_{1}>+2<\mathrm{X}_{1}, \mathrm{X}_{2}>+<\mathrm{X}_{2}, \mathrm{X}_{2}>=2<\mathrm{X}_{1}, \mathrm{X}_{2}>$

This is a contradiction, so that we have $k_{1}=k_{2}$. Since $\operatorname{dim} S=2, p$ is an umbilic point.

Proof of Corollary A.1. By the assumption, for each point $p \in S$, there exit two lightlike line on $S$ which through $p$. By Theorem $\mathrm{A}, S$ is a pseudosphere or a time-like plane, however, a time-like plane does not satisfy the assumption.

Proof of Corollary A.2. It follows from Theorem A that there exists real number $k$ such that $-\nabla_{\mathbf{X}} \xi=k \mathrm{X}$ for any $\mathrm{X} \in T_{p} S$. By the assumption, there exists a non-light-like line $\gamma$ on $S$ through $p$, so that we have $-\nabla_{\gamma^{\prime}} \xi=k \gamma^{\prime}$. Since $\gamma$ is a line, $\sigma\left(\gamma^{\prime}, \gamma^{\prime}\right)=0$. Thus we have

$$
k<\gamma^{\prime}, \gamma^{\prime}>=<k \gamma^{\prime}, \gamma^{\prime}>=<-\nabla_{\gamma^{\prime}} \xi, \gamma^{\prime}>=\sigma\left(\gamma^{\prime}, \gamma^{\prime}\right)=0,
$$

so that $k=0$ because $\gamma$ is non-light-like. Therefore $p$ is a geodesic point. This completes the proof.

Proof of Theorem B. Let $\gamma$ be a light-like line on $S$ through p. By Lemma 2.3, there exists a real number $k$ such that $-\nabla_{\gamma^{\prime}} \xi=k \gamma^{\prime}$. Let $\gamma_{i}(i=1,2)$ be non-light-like planar geodesics. By Lemma 2.4, we have $-\nabla_{\gamma_{i}^{\prime}} \xi=k_{i} \gamma_{i}^{\prime}\left(k_{i} \neq 0\right)$ or $\sigma\left(\gamma_{i}^{\prime}, \gamma_{i}^{\prime}\right)=0$. Thus we distinguish the following three cases.

Case 1) $k_{1} \neq 0$ and $k_{2} \neq 0$.

Assume that $k=0$, so that $-\nabla_{\gamma^{\prime}} \xi=0$. Since $\operatorname{dim} S=2$, there exist real numbers $\lambda, \mu$ such that $\gamma^{\prime}=\lambda \gamma_{1}^{\prime}+\mu \gamma_{2}^{\prime}$. Thus we have

$$
0=-\nabla_{\gamma^{\prime}} \xi=\lambda\left(-\nabla_{\gamma^{\prime}} \xi\right)+\mu\left(-\nabla_{\gamma^{\prime}} \xi\right)=\lambda k_{1} \gamma_{1}^{\prime}+\mu k_{2} \gamma_{2}^{\prime}
$$

Since $\gamma_{1}^{\prime}, \gamma_{2}^{\prime}$ are linearly independent, $\lambda k_{1}=\mu k_{2}=0$, then $\lambda=\mu=0$. This contradicts the fact that $\gamma^{\prime} \neq 0$. It follows that there exist three principal directions $\gamma^{\prime}, \gamma_{1}^{\prime}$ and $\gamma_{2}^{\prime}$, then $p$ is an umbilic point.

Case 2) $\sigma\left(\gamma_{1}^{\prime}, \gamma_{1}^{\prime}\right)=\sigma\left(\gamma_{2}^{\prime}, \gamma_{2}^{\prime}\right)=0$ 
In this case $\left.<-\nabla_{\gamma_{i}^{\prime}} \xi, \gamma_{i}^{\prime}\right\rangle=\sigma\left(\gamma_{i}^{\prime}, \gamma_{i}^{\prime}\right)=0(i=1,2)$ and $-\nabla_{\gamma^{\prime}} \xi=k \gamma^{\prime}$. Since $\gamma^{\prime}, \gamma_{1}^{\prime}, \gamma_{2}^{\prime}$ are linearly independent, there exist non-zero real numbers $\lambda, \mu$ such that $\gamma^{\prime}=\lambda \gamma^{\prime}+\mu \gamma_{2}^{\prime}$. Thus we have

$$
0=<-\nabla_{\gamma_{1}^{\prime}} \xi, \gamma_{1}^{\prime}>=2 \lambda \mu<-\nabla_{\gamma^{\prime}} \xi, \gamma_{2}^{\prime}>=2 \lambda \mu<k \gamma^{\prime}, \gamma_{2}^{\prime}>=2 \lambda \mu k<\gamma^{\prime}, \gamma_{2}^{\prime}>
$$

so that $\left.2 k<\gamma^{\prime}, \gamma_{2}^{\prime}\right\rangle=0$. By the assumption, we have $\left\langle\gamma^{\prime}, \gamma^{\prime}\right\rangle=0$ and $\left\langle\gamma_{2}^{\prime}, \gamma_{2}^{\prime}\right\rangle \neq 0$. It follows from Lemma 2.1 that $\left\langle\gamma^{\prime}, \gamma_{2}^{\prime}\right\rangle \neq 0$. Thus we have $k=0,4$ so that $-\nabla_{\gamma^{\prime}} \xi=0$. For any $\mathrm{X} \in T_{p} S$, there exist real numbers $\nu, \rho$ such that $\mathrm{X}=\nu \gamma_{1}^{\prime}+\rho \gamma^{\prime}$. We have

$$
\sigma(\mathrm{X}, \mathrm{X})=2 \nu \rho \sigma\left(\gamma^{\prime}, \gamma_{1}^{\prime}\right)=2 \nu \rho<-\nabla_{\gamma^{\prime}} \xi, \gamma_{1}^{\prime}>=2 \nu \rho<0, \gamma^{\prime}>=0
$$

This means that $p$ is a geodesic point.

Case 3) $k_{1} \neq 0$ and $\sigma\left(\gamma_{2}^{\prime}, \gamma_{2}^{\prime}\right)=0$.

If $k \neq k_{1}$, we have

$$
\begin{aligned}
& k<\gamma^{\prime}, \gamma_{1}^{\prime}>=<k \gamma^{\prime}, \gamma_{1}^{\prime}>=<-\nabla_{\gamma^{\prime}} \xi, \gamma_{1}^{\prime}> \\
& =<\gamma^{\prime},-\nabla_{\gamma^{\prime}} \xi>=<\gamma^{\prime}, k_{1} \gamma_{1}^{\prime}>=k_{1}<\gamma^{\prime}, \gamma_{1}^{\prime}>
\end{aligned}
$$

It follows that $\left\langle\gamma^{\prime}, \gamma_{1}^{\prime}\right\rangle=0$. This contradicts to Lemma 2.1 , so that $k=k_{1}$. Since $\gamma^{\prime}, \gamma_{1}^{\prime}$ are linearly independent, $p$ is an umbilic point. This completes the proof of Theorem $C$.

Proof of Corollary B.1. Since a non-light-like line on $S$ is a planar geodesic, $S$ is totally umbilic by Theorem B. By exactly the same arguments as those in the proof of Corollary A.2, $S$ is totally geodesic. This completes the proof.

\section{EXAMPLES}

In this section we give some examples which indicate that Theorems $A, B$ and those corollaries are the best possible.

EXAMPLE 4.1. We consider the following parametrized surface in $\mathbb{M}^{3}$

$$
\mathrm{X}(u, v)=\left(\cos u, \frac{1}{\sqrt{2}}(\sin u+v), \frac{1}{\sqrt{2}}(-\sin u+v)\right)
$$

where $|u|,|v|$ are small enough. The surface is a part of a cylinder whose rulings are light-like in $\mathbb{M}^{3}$, so that it has only one light-like line through each point. This example describes that Theorem $\mathrm{A}$ is the best possible.

EXAMPLE 4.2. Since a pseudosphere has just two light-like curve through each point, Corollary A.2 is the best possible. 
EXAMPLE 4.3. We consider the following ruled surface in $\mathbb{M}^{3}$

$$
\mathbf{X}(u, v)=(a(\cos u-v \sin u), b(\sin u+v \cos u), v),
$$

where $a, b>0$ and $a \neq b$. An outside of some closed subsets on the surface has two nonlight-like line through each point. Of course these lines are planar geodesics on the surface, so that Theorem B and Corollary B.1 are the best possible.

\section{REFERENCES}

[1] OGIUE, $\mathrm{K}$ and TAKAGI, $R$ : A Submanifold which contains Many Extrinsic Circles. Tsukuba J. Math. 8 (1984), 171-182

[2] O'NEIL, B : Semi-Riemannian Geometry. Academic Press (1983)

[3] TAKEUCHI, N. : A Sphere as a Surface which contains Many Circles. Journal of Geometry 24 (1985), 123-130

[4] TAKEUCHI, N. : A Surface which contains planar geodesics. Geometriae Dedicata 27 (1986), 223-225

[5] TAKIYAMA, A : Characterizations of a pseudosphere in Minkowski 3-space. Master Thesis of Hokkaido University (1995)

S. Izumiya and A. Takiyama Department of Mathematics, Faculty of Science, Hokkaido University, Sapporo 060, Japan. 